\title{
Delle funzioni regolari in un' area connessa qualsivoglia a distanza finita.
}

\author{
(Di Grorio Ascoli, a Milano.)
}

\begin{abstract}
1. Dirò regolare una funzione $f(x, y)$ dei punti di un'area piana connessa ad uno strato $A$ posta a distanza finita, quando la superficie $A$ possa dividersi in un numero limitato di pezzi $B_{t}(t=1,2, \ldots, m ; m \geqslant 1)$, in ciascuno dei quali la $f(x, y)$ si comporti in una delle quattro note maniere $\left(^{*}\right)$. Ad una tale funzione si accennerà col simbolo (I, II, III, IV). Suppongo poi tra loro sconnesse due aree $B_{\sigma}$ e $B_{\tau}\left(\sigma_{<}>\tau\right)$, nelle quali la data funzione è della stessa specie, laddove l'ente $A$ cade nel primo quadrante e la $f(x, y)$ non raggiunge dei valori nulli o negativi. Mediante una scelta opportuna degli assi si pud soddisfare alle due ultime condizioni.

Consideriamo ora il modo di comportarsi della funzione data da ambo le parti del ramo primitivo e non decrescente $L-\varepsilon\left({ }^{* *}\right)$, essendo $L$ un elemento che separa le due aree $B_{\sigma}$ e $B_{\tau}(\sigma \geq \tau)$, mentre non si può assegnare un ramo primitivo, di cui l'ente $L$ sia una parte, il quale appartenga in pari tempo alle due linee $C_{B_{\sigma}}$ e $C_{B_{\tau}}$. Con la notazione $L-\varepsilon$ indico, come al solito, ' la linea $L$, quando si trascuri ad ognuno dei suoi estremi un tratto piccolo quanto si vuole. L'ente $L$ potrà anche essere un tratto di retta parallelo ad uno dei due assi, come è manifesto; però in seguito si accennerà in modo speciale a questo fatto.

E subito visto che la linea $L$ non limiterà due aree in ognuna delle quali la funzione studiata è di specie pari od impari, mentre per ogni punto $h$
\end{abstract}

(*) Vedi tatta la mia Memoria inserita nel volume precedente di questi Ann

(**) Vedi il $\$ 1$ del n. 5 della stessa Memoria. 
dell' ente $L-0$ esce una linea $f(x, y)=C$, la quale, considerata nelle vicinanze del punto $h$ e da ambo le parti, si projetta semplicemente sopra uno degli assi coordinati. Ciò si verifica, perchè nell'ipotesi opposta la linea $f(x, y)=C$ uscente dall'elemento $h$ sarebbe da una parte e dall'altra della curva $L-\varepsilon$ ognora crescente oppure decrescente all' aumentare della ascissa, laddove ogni elemento $f(x, y)=C$ si projetta in modo semplice sopra uno degli assi ed in questo caso quindi anche sull'altro; la $f(x, y)$ sarebbe di conseguenza della stessa specie in $B_{\sigma}$ ed in $B_{\tau}$, la qualcosa è contraria all'ipotesi.

Se di più si ammette che la funzione $f(x, y)$ sia crescente dal primo al secondo estremo della linea $L$, daremo origine al quadro in appresso I), di cui il significato è palese, purchè si abbiano presenti le osservazioni che seguono.

Con la notazione $+L$ indicherò un ramo primitivo ognora crescente con l'ascissa, laddove il simbolo $-L$ accenna pure ad un elemento primitivo, il quale però decresce all'aumentare della variabile $x$.

Il segno $+L^{(+)}$dirà che lungo l'ente $+L$ è fissata una successione continua di valori ognora crescenti insieme all' ascissa $x$ ed analogo significato va attribuito alla notazione $-L^{(+)}$. Con la scrittura $I I,+L^{(+)}$, I accenno poi al fatto che alla destra dell' cnte $+L^{(+)}$, percorso in guisa, che la funzione $f(x, y)$ aumenti, la espressione stessa $f(x, y)$ è di prima specie e di seconda alla sinistra. Ed ora parmi facile l'intelligenza del quadro in discorso.

$$
\begin{array}{ll}
\text { 1) } \mathrm{II},+L^{(+)}, \mathrm{I} ; & \text { 2) } \mathrm{I},+L^{(+)}, \mathrm{II} \text {; } \\
\text { 3) } \mathrm{I},+L^{(+)}, \mathrm{IV} ; & \text { 4) } \mathrm{IV},+L^{(+)}, \mathrm{I} \text {. }
\end{array}
$$

Chiamo $B_{\sigma}$ quella parte dell' area $A$ in cui la $f(x, y)$ è di una sola specie e che giace alla destra dell'elemento $+L$, percorso in guisa, che l'ascissa cresca. L'ente $B_{\tau}$ cada invece alla sinistra del ramo primitivo $+L$.

Nella figura 1) la funzione $f(x, y)$ è di seconda specie in $B_{\tau}$ e di prima in $B_{\sigma}$, e da ogni punto dell' arco $L-0$ esce una linea $f(x, y)=C$, che ha ivi un massimo rispetto all'asse $X$, sopra il quale si projetta semplicemente. Nel disegno successivo l'insieme $f(x, y)$ che passa per un elemento della curva $L-\varepsilon$ ha in quest' ultimo un minimo relativamente alla retta $y=0$, su cui si specchia in maniera univoca. Se poi la $f(x, y)$ è di prima specie in $B_{\tau}$ e di quarta in $B_{\sigma}$, da ogni punto della curva $L-\varepsilon$ esce una linea $f(x, y)=C$, la quale ha un massimo rispetto alla retta $x=0$, sulla quale si projetta in modo semplice, mentre si verifica l'opposto, se la $f(x, y)$ è di quarta specie in $B_{\tau}$ e di prima in $B_{\sigma}$. 
La funzione contemplata non pud essere di terza specie da una parte dell' ente $L-0$, perchè essa è crescente lungo $+L$; il quadro I è dunque completo nella ipotesi che la linea $f(x, y)=C$ si projetti in modo semplice sopra uno degli assi nelle vicinanze del punto in cui interseca l'ente $L$.

Se l'elemento $L$ fosse un tratto parallelo all' asse $Y$ non potrebbero verificarsi che gli eventi 1) e 2), e gli altri due soltanto quando l'intervallo fosse parallelo alla retta $y=0$.

Sia ora la funzione $f(x, y)$ decrescente lungo il ramo $+L$, laddove ogni linea $f(x, y)$, la quale contiene un punto dell' elemento $L-0$ ha un'imagine semplice sopra uno degli assi coordinati. In tale ipotesi daremo origine al quadro:

II)
1) III, $+L^{(-)}$, II;
2) II, $+L^{(-)}$, III;
3) IV, $+L^{(-)}$, III,
1) III, $+L^{(-)}$, IV.

Nelle figure 1) e 2) l'elemento $L$ potrà venir sostituito da un intervallo di retta parallelo all' asse $X$, all' altro nelle ultime due.

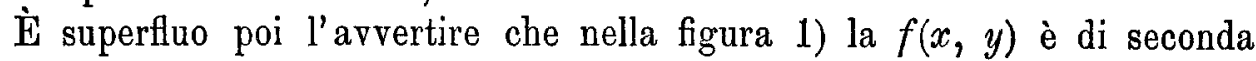
specie alla destra dell' ente $+L^{(-)}$percorso in guisa, che la funzione data cresca lungo il medesimo.

Quando si supponga la $f(x, y)$ costante lungo $+L$, la qual cosa si esprimerà con la notazione $+\bar{L}$, avremo lo specchio:
1) II, $+\bar{L}, \mathrm{IV}$
2) IV, $+\bar{L}$, II.

È chiaro che in questo quadro l'ente $+L$ non può ridursi ad un tratto parallelo ad uno degli assi coordinati. Nella prima figura dello stesso specchio ogni punto della linea $L-0$ è un minimo della espressione $f(x, y)$; nella seconda un massimo. Si suppone che nel disegno 1) la $f(x, y)$ sia di quarta specie alla destra dell' ente $L$ percorso in guisa che l'ascissa cresca.

2. Sia adesso la linea $L$ sempre decrescente e la funzione $f(x, y)$ cresca lungo la medesima all' aumentare dell' ascissa. In tale ipotesi le cose stanno come lo indica la tabella:

$$
\begin{aligned}
& \text { 1) I, }-L^{(+)}, \mathrm{IV} ; \quad \text { 2) } \mathrm{IV},-L^{(+)}, \mathrm{I} \text {; } \\
& \text { 3) IV }-L^{(+)}, \mathrm{III}, \quad \text { \&) III, }-L^{(+)} \text {, IV. }
\end{aligned}
$$

Nei due primi disegni il ramo $L$ potrebbe venir surrogato da un segmento parallelo all' asse $X$, negli altri da un tratto normale a quest'ultimo. 
Quando poi la $f(x, y)$ decresce lingo $-L$ aumentando l'ascissa, avremo le figure dello specchio:

V)

$$
\begin{array}{ll}
\text { 1) II, }-L^{(-)}, \mathrm{I} ; & \text { 2) I, }-L^{(-)}, \text {II } \\
\text { 3) III }-L^{(-)}, \mathrm{II} ; & \text { 4) II, }-L^{(-)} \text {, III. }
\end{array}
$$

Nel caso che la funzione contemplata fosse costante nell' ente $-L$, andrebbero considerati i due disegni che seguono, nel primo dei quali la $f(x, y)$ è di prima specie in $B_{*}$. 亡̀ chiaro quindi che nella figura 1) la nostra funzione arrà un minimo in ogni punto dell' arco $L-0$, laddove nell' altra essa arrà un massimo,

$$
\text { 1) I, }-\bar{L}, \text { III ; III, }-\bar{L}, \text { I. }
$$

Osservando con attenzione le tabelle I), II), IV), V) e la figura in appresso, troveremo una regola assai semplice per compendiare i risultati ottenuti

$$
\begin{aligned}
& -+,+Y,++ \\
& -X, \quad 0, \quad+X \\
& --, \quad-Y, \quad+-
\end{aligned}
$$

Se io percorro il verso positivo dell' asse $Y$, ho alla destra il primo quadrante ed alla sinistra il secondo, ed analoghe osservazioni si possono fare rispetto alle altre direzioni degli assi.

Ciò premesso, se il ramo $L$ separa un'area in cui la $f$ è di prima specie da un'altra nella quale è di seconda e se la prima è alla sua destra, quando si percorra in guisa, che la $f(x, y)$ aumenti, per ogni punto della linea $L-\varepsilon$ esce una varietà $f(x, y)=C$, la quale si projetta semplicemente sull' asse $X$ ed ha in esso un massimo ossia accenna al verso positivo dell' asse $Y\left({ }^{*}\right)$ $[I), 1) ; \mathrm{V}), 1)]$.

Nel caso contemplato diremo che la $f(x, y)$ si comporta in modo naturale dalle due bande della linea $L-0$.

Se all'incontro il ramo $L$ limitasse un elemento in cui la $f(x, y)$ è di prima specie da un altro nel quale è di seconda, e se il primo si trovasse alla sinistra della varietà $L$ percorsa in guisa, che la funzione $f(x, y)$ cresca, per ogni punto della linea $L-\varepsilon$ escirebbe un insieme della forma $f(x, y)=C$, che si specchia in maniera semplice sulla retta $y=0$ ed ha in esso un minimo, accenna cioè al verso negativo dell' asse $Y$ [L), 2); V), 2)].

$\left(^{*}\right)$ Mi sia concesso l'uso di questa frase, il eui significato è palese. 
In tale ipotesi diremo che la funzione contemplata si contiene in modo non naturale dalle due parti dell'ente $L$.

Se poi osserviamo con attenzione le coppie di disegni

$$
\begin{gathered}
[(\mathrm{II}), 1) ; \mathrm{V}), 3)]-[(\mathrm{II}), 2) ; \mathrm{V}), 4)] ; \quad[(\mathrm{II}), 3) ;(\mathrm{V}), 4)]-(\mathrm{II}), 4) ;(\mathrm{V}), 3)] ; \\
[\mathrm{I}), 3) ;(\mathrm{IV}), 1)]-[(\mathrm{I}), 4) ;(\mathrm{IV}), 2)]
\end{gathered}
$$

avvertiremo tosto la verità della proposizione:

Ogni qualvolta la funzione studiata si comporta nel modo naturale dalle due parti dell'elemento $L-0$ guardato nel verso in cui la $f(x, y)$ cresce, la linea $f(x, y)=$ cost. si projetta nelle vicinanze del punto in cui incontra $l$ 'arco $L$ semplicemente sulla normale a quell' asse che divide $i$ quadranti analoghi ed accenna a quel verso di quest'ultimo rispetto al quale si ha la disposizione naturale. Se poi la $f(x, y)$ si contiene in modo non spontaneo dalle due bande dell'ente $L-0$, l'elemento $f(x, y)=C$ ha un' imagine semplice sull'asse or ora indicato ed accenna a quel verso dell'altro, che è contrario alla maniera spontanea.

In ciascuno dei quadri I), II), IV), V) l'elemento primitivo $L$ potrebbe esser sostituito da un ramo semplice, il quale però non conterrebbe che un numero limitato $(\supseteq 0)$ di tratti paralleli ad uno degli assi soltanto. Così, ad esempio, nei due primi disegni dello specchio I) l'ente $L$ potrebbe esser dotato di uno o più segmenti paralleli all'asse $Y$ ma non all'altro, laddove negli altri due $\mathrm{i}$ tratti in discorso dorrebbero essere paralleli alla retta $y=0$.

3. Consideriamo ora il caso che nei pressi di un punto dell'elemento $L-0$ la linea $f(x, y)=C$ non si projetti semplicemente sopra veruno dei due assi coordinati. In tale ipotesi daremo origine al quadro:
$\left.I_{1}\right)$
1) II, $+L^{(+)}$, IV;
2) $\mathrm{IV},+L^{(+)}, \mathrm{II}$;
3) IV $+L^{(-)}, \mathrm{II}$;
4) II, $+L^{(-)}$, IV;
5) I, $-L^{(+)}$, III
c) III, $-L^{(+)}, \mathrm{I}$;
7) III, $-L^{(-)}, \mathrm{I}$;
s) I, $-L^{(-)}$, III.

In questo caso non può tenersi parola nè di massimo nè di minimo rispetto ad uno degli assi coordinati, perchè la linea $f(x, y)=C$ non si projetta semplicemente sopra veruna delle due rette $x y=0$ nelle vicinanze del punto in cui incontra il ramo $L-0$. Facendo attenzione all'ultimo specchio possiamo quindi enunciare la proposizione: 
L'ente $f(x, y)=C$ accenna ognora nei pressi del punto in cui incontra la linea $L$ a quel verso di quest'ultima lungo il quale la funzione $f(x, y)$ cresce, se quest' ultima è disposta nel modo naturale rispetto all'elemento $L$, in caso opposto indica il verso contrario.

Suppongo adesso che lungo il ramo $+L$ la $f(x, y)$ non sia ognora crescente o decrescente all' aumentare dall' ascissa oppure sempre costante. In altre parole, ammetto che il tratto $L$ si possa dividere in un numero limitato di parti $m_{1}, m_{2}, \ldots, m_{p}(p>1)$, procedendo sopra $L$ in guisa, che l'ascissa cresca, per modo, che in due successive la $f(x, y)$ non si comporti nella stessa maniera.

Nella nuova ipotesi la $f(x, y)$ non potrà essere che di seconda specie da una parte dell'ente $+L$ e di quarta dall'altra dando così origine allo specchio:
$\mathrm{II}_{4}$ )
1) II, $+L, \mathrm{IV}$;
\&) IV $+L$, II,

nel quale il simbolo $+L$ accenna al fatto che la funzione contemplata non si comporta sempre nella stessa guisa lungo l'arco $L$, laddove la $f(x, y)$ è di specie quarta in $B_{\text {s }}$ nel primo disegno e di seconda nell'altro. Circa poi alla maniera di contenersi della funzione $f(x, y)$ dalle due parti dell'ente $L$ giova rammentare l'ultima proposizione aggiungendovi le osservazioni che seguono.

Intorno ad un tratto $q(\geqslant 0)$ di minimo della $f(x, y)$ considerata nella sua dipendenza dalla curva $L-0$ esiste nell' ipotesi del disegno $\left.\left.\left[\mathrm{II}_{1}\right), 1\right)\right]$ una varieta di linee chiuse, che tendono all' intervallo $q$, lungo ciascuna delle quali la $f(x, y)$ si mantiene costante. L'ente $q$ può quindi considerarsi come un minimo anche per la funzione $f(x, y)$ considerata nella sua dipendenza dai punti dell' area $A$. Se poi sempre nella ipotesi del disegno $\left[\mathrm{II}_{4}\right), 1$ )] l'intervallo $q$ fosse un massimo della nostra funzione considerata semplicemente lungo $L-0$, ogni punto del tratto $q-0$ sarebbe un minimo per la funzione data, nè la linea $f(x, y)=C$ si comporterebbe nel modo detto poco fa nei pressi del segmento $q$, tenderebbe bensì con una sua parte al medesimo, la quale però è formata da due tratti sconnessi tra loro.

Analogamente, guardando il disegno $\left.\left.\left[\mathrm{II}_{1}\right), 2\right)\right]$, avvertiremo tosto che, se il tratto $q(\geqslant 0)$ è un massimo per la $f(x, y)$ nella sua dipendenza dalla curva $L-0$, sarà altresi tale per la funzione data ed esisterà un insieme di linee chiuse, che si riducono all'ente $q$, lungo ciascuna delle quali la $f(x, y)$ non muta di valore. Se all' incontro, stando ognora lo stesso disegno, il tratto $q(\geq 0)$ 
è un minimo della $f(x, y)$ rispetto al tratto $L-0$, ogni punto dell'ente $q-0$ è un massimo della $f(x, y)$.

Quando l'elemento $L$ sia decrescente, si dovrà contemplare il quadro:
$\mathrm{III}_{4}$ )
1) I, $-L$, III;
2) III, $-L, \mathrm{I}$,

circa al quale vanno fatte delle osservazioni analoghe a quelle relative allo specchio $\mathrm{II}_{1}$ ).

Possiamo quindi enunciare $\mathrm{i}$ due teoremi:

Un tratto $q(\geqslant 0)$ di minimo della $f(x, y)$ nella sua dipendenza dall'arco $+L-0$ è pure tale per la funzione data, se quest'ultima si contiene in modo naturale dalle due bande dell' ente $+L$ guardato nel verso in cui cresce l'ascissa, ed una asserzione analoga si faccia di un massimo, essendo però inverso il modo di comportarsi della $f(x, y)$ dalle due parti dell'ente $+L$.

Il secondo teorema è conforme al precedente, quando in esso si muti l'elemento $+L$ nell' altro $-L$.

4. Studiato il modo di contenersi della funzione $f(x, y)$ dalle due parti della linea $L-0$, la quale appartenga in pari tempo alle due curve $C_{B_{\sigma}}$ e $C_{B_{\tau}}(\sigma \geq \tau)$, vediamo adesso come essa si comporti ai suoi estremi. Potranno darsi due eventi, il termine contemplato, che dirò $c$, dell' ente $L$ è soltanto l'estremo di due rami primitivi delle linee $C_{B_{5}}$ e $C_{B_{*}}$, i quali considerati insieme non formano al certo un elemento primitivo, oppure tal fatto non ha luogo. Se mi muovo nella prima ipotesi lungo $L$ verso $\partial$ in guisa ohe l'ascissa cresca, ho dall'una parte, e precisamente alla destra, l'area $B_{\boldsymbol{\sigma}}$ e dall' altra l'elemento $B_{\tau}$, mentre lo stesso avviene se continuo il mio cammino dopo il punto $c$ nella curva $C_{B_{\sigma}}$. Chiamo poi per semplicità $l$ una parte connessa del ramo $L$ dotata di un termine in $c$ e con $l_{1}$ un altro tratto primitivo connesso delle due curve $C_{B_{\sigma}}$ e $C_{B_{\tau}}$ che è unito al primo solamente mediante il punto $c$. $\dot{\mathrm{E}}$ chiaro che si può ammettere che lungo il segmento $l$ verso $c$ la funzione $f(x, y)$ sia ognora crescente o decrescente oppure costante, e la stessa cosa si dica dell' ente $l_{1}$.

Ora, se io posso tracciare pel punto $O$ dell" area $A-0$ due rami primitivi, che insieme ad un terzo non uscente dal medesimo mi limitino una parte semplicemente connessa $B$ dell'elemento $A$, e se la $f(x, y)$ non raggiunge in $B$ dei valori più grandi che in $O$, diremo che la funzione data ha un massimo parziale in $O$ rispetto alla superficie $B$. Analogamente si definisce il minimo parziale. 
Ciò premesso, se gli enti $l$ ed $l_{1}$ sono crescenti andando verso $c$, daremo origine alla tabella $\left({ }^{*}\right)$ :
1) $\mathrm{II}, \overline{l_{1}^{(+)}, \mathrm{I}, \quad+l^{(+)}} \mid, \mathrm{II}$;
2) IV $\left.\bar{\mp} \overline{l_{1}^{(+)}}, \overline{\mathrm{I}}, \quad+l^{(+)}\right), \mathrm{IV}$;
3) I, $\left.+l_{1}^{(+)}, \mathrm{II},+l^{(+)}\right), \mathrm{I}$;
4) IV, $\overline{+l_{1}^{(+)}}, \mathrm{II},+l^{(+)} \mid, \mathrm{IV}$;
5) I, $+l_{1}^{(+)}, \mathrm{IV},+l^{(+)} \mid, \mathrm{I}$;
6) II, $\overline{+l_{1}^{(+)}, \mathrm{IV}} \overline{,+l^{(+)}} \mid$, II;
7) III, $\overline{+l_{1}^{(-)}, \mathrm{II},+l^{(-)}}$, III;
8) IV, $\overline{+l_{1}^{(-)}, \mathrm{II},+l^{(-)}}, \mathrm{IV}$;
9) II, $\overline{+l_{1}^{(-)}, \text {III },+l(-)} \mid$, II
10) IV, $+l_{1}^{(-)}$, III, $+l^{(-)} \mid, I V$;
11) II, $\overline{+l_{1}^{(-)}}, \mathrm{IV},+l^{(-)} \mid, \mathrm{II}$;
12) III, $\overline{+l_{1}^{(-)}, \mathrm{IV},+l^{(-)}} \mid$, III;
13) IV, $\overline{+l_{1}^{(+)}, \mathrm{II},+l^{(-)}}$, IV;
14) II, $+l_{1}^{(+)}, \mathrm{IV},+l^{(-)} \mid$, II.

Ed alle due ultime figure si potrebbero aggiungere quelle che si ottengono scambiando gli enti $+l_{1}^{(+)},+l^{(-)}$per ordine negli altri $+l_{1}^{(-)},+l^{(+)}$.

In ognuno dei primi sei disegni l'ente $c$ è un massimo parziale rispetto ad un'area di semplice connessione limitata in parte dalle curve $l$ ed $l_{1}$ e sita nel terzo quadrante di $c$, nelle sei successive all'incontro un minimo.

Allo specchio ora contemplato vanno aggiunti altri tre, i quali nascono supponendo che $\mathrm{i}$ due rami $l$ ed $l_{4}$ cadano successivamente ed in pari tempo nel quarto, primo e secondo quadrante. Questi quattro quadri si indicheranno per ordine con le notazioni $I_{3}, I_{1}, I_{1}, I_{2}$.

Giova contemplare anche la tabella che segue, quando $i$ due elementi $l$ ed $l_{1}$ non cadono in uno stesso quadrante.
1) II, $\left.\mp \overline{l(+)}\right|^{c}, \mathrm{I}, \bar{c}=l_{1}^{(-)}, \mathrm{II}$;
2) I, ${\overline{+l^{(+)}}}^{0}, \mathrm{II}, \stackrel{\overline{-l_{1}^{(-)}}}{\mathrm{C}} \mathrm{I}$;
3) IV, $\left.\overline{+l(+)}\right|^{0}, \mathrm{I}, \quad{ }^{c}=l_{1}^{(+)}, \mathrm{IV}$;
4) I, $\overline{+\left.l^{(+)}\right|^{c}}, \mathrm{IV}, \stackrel{c}{\bar{c}-l_{1}^{(+)}}, \mathrm{I}$;
5) IV, $\left.\overline{+l(-)}\right|^{c}$, III, ${ }^{c}=l_{1}^{(+)}$, IV;
6) III, $\left.\overline{+l^{(-)}}\right|^{c}, \mathrm{IV}, \overline{-l_{1}^{(+)}}$, III;
7) III, $\left.\overline{+l(-)}\right|^{c}$, II, ${ }^{c} \overline{-l_{2}^{(-)}}$, III;
8) II, $\overline{+l(-)}^{c}$, III, $\overline{-l_{1}^{(-)}}$, II.

Se si osserva che $i$ numeri $1,2,3,4$ dànno luogo alle sei combinazioni 1,$2 ; 1,3 ; 1,4 ; 2,3 ; 2,4 ; 3,4$, avvertiremo tosto che si possono formare altre cinque tavole analoghe alla precedente, che torna acconcio indicare col

(*) Il significato di questa tabella e delle successive mi sembra chiaro, quando si osservi che con le notazioni $\ldots, \ldots,-1,1$ accenno per ordine ai quattro quadranti. 
simbolo $I_{3,4}$. Di conseguenza, alle altre si accennerà coi segni $I_{1,2}, I_{1,3}, I_{1,4}$, $I_{2,3}, I_{2,4}$, dalle quali però vanno tolti $i$ quadri $I_{1,3}, I_{2,4}$, poichè in essi gli elementi $l$ ed $l_{1}$ formano un solo ramo primitivo, il che va per ipotesi escluso.

Per le ricerche precedenti possiamo quindi enunciare il teorema $\left(^{*}\right)$ :

La funzione contemplata non ammette un massimo od un minimo relativo in un punto dell' area $A-0$, nel quale si incontrano soltanto due rami primitivi della curva $\frac{1}{2}\left(\Sigma C_{B_{s}}-C_{A}\right)$, che non formano un ramo semplice. Può all' incontro essere dotata in esso di un massimo o di un minimo parziale.

In alcune delle figure di ciascuno dei quadri $I_{1,2}, I_{1,4}, I_{2,3}, I_{3,4}$ uno soltanto degli enti $l$ ed $l_{1}$ potrebbe cadere sull' asse $Y$ oppure sull'altro. Cosi, ad esempio, nella figura 1) dello specchio $I_{3,4}$ soltanto una delle linee $l_{1}$ ed $l$ a piacere potrebbe ridursi ad un intervallo della retta $x=0$, ed in allora si otterrebbe un caso limite di una delle figure del disegno $I_{4}$ oppure dell'altro $I_{3}$ per ordine.

Contempliamo ora il caso che la $f(x, y)$ sia costante lungo uno soltanto degli elementi $l$ ed $l_{1}$, chè per le nostre ipotesi circa alla medesima non può esser tale in amendue. Stando in tal modo le cose, $i$ due enti $l$ ed $l_{1}$ cadranno di necessità in uno stesso quadrante, poichè, se ciò non avesse luogo, ossia se uno degli elementi $l$ fosse in un quadrante e l'altro nel contiguo la $f(x, y)$ dovrebbe essere della stessa specie da una banda e dall'altra della curva $l+l_{1}$. Infatti, se fosse, ad esempio $\left.\overline{+\bar{l}}\right|^{c}, \mid \overline{-l} l_{1}^{(-)}$, la $f(x, y)$ non potrebbe essere nè di prima nè di terza specie nelle vicinanze del punto $c$ in quanto non muta nell' arco $+l$ sito nel terzo quadrante, non sarebbe poi di quarta perchè la $f(x, y)$ decresce lungo $-l$, al crescere della variabile $x$. Adunque, vicino al punto $c$ dovrebbe essere solamente di seconda, la qual cosa contrasta con la ipotesi che in due aree contigue $B_{\tau}$ e $B_{\tau}$ la $f(x, y)$ sia di specie diversa.

Supposto quindi che le curve $l$ ed $l_{1}$ cadano nel terzo quadrante, si dovrà contemplare il quadro:
1) II, $\left.\overline{+l_{1}{ }^{(+)}, \mathrm{IV},+\bar{l}}\right|^{c}, \mathrm{II}$;
2) II, $\left.\overline{+\bar{l}_{1}, \mathrm{IV},+l^{(+)}}\right|^{c}, \mathrm{Ir}$;
3) IV, $+l_{1}^{(-)}, \mathrm{II},+\left.\bar{l}\right|^{c}, \mathrm{IV}$;
4) $\mathrm{IV}, \overline{+\bar{l}_{1}}, \mathrm{II},+\left.l^{(l+)}\right|^{c}, \mathrm{IV}$;
5) IV, $\left.\overline{+l_{1}^{(-)}, \mathrm{II},+\mathrm{l}}\right|^{c}, \mathrm{IV}$;
e) IV $\overline{+\bar{l}_{1}, \mathrm{II},+l^{(-)}} \mid \mathrm{IV}$;
2) II, $\left.\overline{+l_{1}^{(-)}, I V,+\vec{l}}\right|^{c}, \mathrm{II}$;
8) II, $\left.\overline{+\bar{l}_{1}, \mathrm{IV},+l^{(-)}}\right|^{c}$, II.

(") Vedi l'ultimo paragrafo della precedente Memoria. 
Se indico questo quadro con la notazione $S_{3}$, sarà conveniente accennare a quelli che si riferiscono nello stesso modo agli altri tre quadranti coi simboli $S_{4}, S_{1}, S_{2}$ girando da sinistra a destra.

Per ultimo, osservo che nessuno dei due elementi $l$ ed $l_{1}$ del quadro $S_{t}(t=1,2,3,4)$ può ridursi ad un tratto parallelo ad uno degli assi. Quanto al ramo in cui la $f(x, y)$ è costante la cosa è manifesta, circa all'altro giova rammentare che la funzione contemplata non è dalle sue due parti di specie contigue.

5. Consideriamo ora un punto $O$ dell' area $A-0$, nel quale concorrono più di due rami primitivi della curva $\frac{1}{2}\left(\Sigma C_{B_{s}}-C_{A}\right) \equiv D$. Se contemplo uno di questi ultimi avverto subito che la $f(x, y)$ si comporta in maniera diversa dalle sue due bande, perchè non si possono assegnare per jpotesi due aree tra loro sconnesse $B_{\sigma}$ e $B_{\tau}(\sigma \geq \tau)$ per modo, che la $f(x, y)$ si contenga egualmente in entrambe.

A portare un po'di luce circa il modo di comportarsi della funzione $f(x, y)$ intorno all'elemento $O$ giovano le figure seguenti, nelle quali secondo la fatta convenzione la linea $l_{1}$ è alla sinistra dell'ente $l$.
1) $\left.\right|_{0}+l_{1}^{(+)}, 1,+l^{(+)}$;
2) $1+l_{1}^{(+)}$, II,$+l^{(+)}$;
3) $1+l_{1}^{(+)}, \mathrm{IV},+l^{(+)}$;
1) $\left.\right|_{0}+l_{1}^{(-)}$, II, $+l^{(-)}$;
5) $1+l_{1}^{(-)}$, III, $+l^{(-)}$;
6) ${ }_{0} \pm l_{1}^{(-)}, \mathrm{IV},+l^{(-)}$;
$\therefore$ I $+l_{1}^{(+)}, \mathrm{II},+l^{(-)}$;
s) $1+l_{1}^{(+)}, \mathrm{IV},+l^{(-)}$;
9) $1+l_{1}^{(-)}, \mathrm{II},+l^{(+)}$;
10) $\left.\right|_{0}+l_{1}^{(-)}, \mathrm{IV},+l^{(+)}$;
11) ${ }_{0}+l_{4}^{(+)}$, II, $+\tau$
12) ${ }_{0} \pm l_{1}^{(+)}, \mathrm{IV},+\bar{l}$;
13) $\left.\right|_{0} \pm l_{1}^{(-)}, \mathrm{II},+\bar{l}$;
14) $\underset{o}{1}+l_{1}^{(-)}, \mathrm{IV},+\bar{l}$
15) $\left.\right|_{0} \pm \bar{l}_{1}, \quad$ II,$+l(+)$;
16) $1+\bar{l}_{1}, \quad$ IV,$+l^{(+)}$;
17) $I_{0} \pm \bar{l}_{1}, \mathrm{II},+l^{(-)}$;
18) ${ }_{0}+\bar{l}_{1}, \quad$ IV,$+l^{(-)}$.

Nei disegni 1), 2) e 3) del quadro precedente abbiamo un minimo della funzione $f(x, y)$ in $O$ considerata nella sua dipendenza dai punti di un'area elementare limitata in parte dagli enti $l$ ed $l_{\mathrm{f}}$ e da un'altra linea che cade nel primo quadrante dell'elemento 0 . Nelle tre figure successive all' incontro si ha un massimo. Nei disegni 1),5), 7), 10), ad esempio, il ramo $l_{1}$ potrebbe cadere sull'asse $Y$, laddove l'altro $l$ potrebbe adagiarsi sulla retta $y=0$. 
Al quadro ora contemplato sono da aggiungere altri tre, i quali nascono nella ipotesi che i nostri due rami cadano per ordine nel secondo, terzo e quarto quadrante. Ed è manifesto che in taluni dei disegni di questi quadri amendue gli enti $l$ ed $l_{1}$ potrebbero cadere sull' asse $X$ od $Y$ uno soltanto ed anche nessuno dei due.

6. Giovandosi degli specchi di cui è parola nel paragrafo precedente risulta facile lo studio del modo di comportarsi della funzione data nei pressi di un punto $O$ della linea $D \equiv \frac{1}{2}\left(\sum_{i} C_{B_{s}}-C_{A}\right)$, nel quale concorrono $m(>2)$ rami primitivi.

Suppongo prima che in $O$ non cada un massimo o minimo parziale nè uno relativo della funzione $f(x, y)$ e che dal medesimo non esca un ramo in cui la $f(x, y)$ si mantiene costante od un tratto rettilineo parallelo ad uno degli assi. Nella fatta jpotesi avverto subito che nell' interno del primo quadrante del punto $O$ e nelle sue immediate vicinanze si possono verificare soltanto i quattro casi

$$
\begin{aligned}
& \text { 1) II, IV, II, IV }, \ldots, \mathrm{IV} ; \quad \text { 2) II, IV, ... II; s) IV, II, IV,..., II; } \\
& \text { 4) IV, II, .., IV, }
\end{aligned}
$$

quando si faccia astrazione dalle due aree $B$, in una delle quali penetra la parallela all'asse $Y$, mentre nell'altra si interna la parallela alla retta $y=0$ uscente dall' elemento 0 .

Il primo evento ed il terzo si verificano quando da $O$ esce un numero impari $(\supseteq 3)$ di rami primitivi siti entro il primo quadrante, gli altri se il numero degli elementi i quali hanno un estremo in $O$ è pari. $E$ se esso è eguale a due, la $f(x, y)$ è in un' area elementare sita nel primo quadrante del punto $O$ di specie II o IV. Analogamente si dica della seconda, terza e quarta regione rispetto alle specie I, III; II, IV; I, III per ordine.

Se due rami successivi $l$ ed $l_{1}$ della curva $D$ uscenti dal punto $O$ cadono entro due quadranti distinti e contigui, la $f(x, y)$ potrà essere in un'area elementare limitata in parte dagli enti $l$ ed $l_{1}$ di cui il contorno in $O$ forma un angolo minore di $180^{\circ}$ di due specie soltanto, perchè essa non ha per dato in $O$ alcun minimo o massimo parziale o relativo. Così, ad esempio, si ha

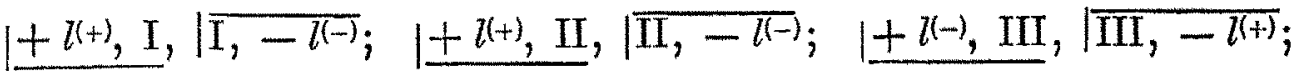

$$
\begin{aligned}
& \text { }+l^{l(-)}, \mathrm{IV}, \mid \mathrm{IV},-l(+) .
\end{aligned}
$$


Se $\mathrm{i}$ due elementi successivi $l$ ed $l_{1}$ cadessero entro due quadranti opposti, poniamo nel primo e terzo, e se l'area $B$ limitata in parte dai medesimi non avesse alcun punto entro la quarta regione dell'ente $O$ ed ad esso vicinissimo, la funzione $f(x, y)$ non potrebbe avere un minimo in $O$ nella sua dipendenza dalla curva $l+l_{1}$, essendo in pari tempo di quarta specie in $B$. Ciò si verifica, perchè nel caso opposto la funzione contemplata avrebbe un minimo parziale in $O$. Così pure, la $f(x, y)$ non potrebbe avere un massimo in $O$ rispetto all'ente $l+l_{1}$, essendo di quarta specie in un'area $B$ terminata in parte ad esso ente, la quale non contiene vicino ad $O$ dei punti siti entro il secondo quadrante di quest'ultimo. Analoghe riflessioni vanno fatte nella ipotesi che $\mathrm{i}$ due elementi $l$ ed $l_{1}$ appartengano alla seconda $\mathrm{e}$ quarta regione dell'elemento 0 . Tolte queste restrinzioni la $f(x, y)$ potrebbe contenersi comunque nell' ente $B$, per quanto è possibile, ammesso che quest' ultimo sia limitato in parte dalla curva $l+l_{1}$ sita entro due regioni opposte.

Se poi l'angolo in $O$ formato dalle linee $l$ ed $l_{1}$ considerate come appartenenti al contorno $C_{B}$ fosse maggiore di $180^{\circ}$ gioverebbe rammentare le osservazioni dell'ultimo paragrafo della Memoria precedente.

Poniamo adesso che intorno al punto $O$ cadano dei massimi e minimi parziali rispetto ad alcune aree $B$ convergenti in esso, ma non un minimo o massimo relativo, nè un ramo in cui la $f(x, y)$ si mantiene costante. Ciò premesso, è evidente che un minimo parziale della $f(x, y)$ non può essere contiguo ad un massimo pure parziale. I casi poi che si possono offrire in questa ricerca, sono oltre ogni dire svariati, come tosto si avverte, e d'altra parte riesce assai facile l'addurre quanti esempi si vogliono. To accennerò soltanto al seguente che si deduce tosto dai nostri quadri

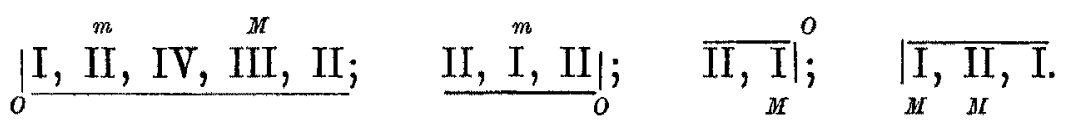

Nel caso contemplato dal punto $O$ escono quattro rami di linea $f(x, y)$ $=$ cost., due nel primo quadrante (IV, II), uno nel terzo (II) ed uno nel quarto (I).

La funzione $f(x, y)$ potrà esser dotata anche di un massimo o minimo relativo in $O$, come nei due esempi che seguono

$$
{ }_{0}^{\mid I I I},\left.\underline{\text { IV }}\right|_{0},\left.\overline{\mathrm{I}}\right|^{0},|\overline{\mathrm{II}} ; \quad|_{0}|\underline{\mathrm{I}}, \underline{\mathrm{II}}|_{0},\left.\overline{\mathrm{III}}\right|^{0}, \mid \overline{\mathrm{IV}} .
$$


Tanto nella jpotesi del massimo relativo che in quella del minimo si può assegnare una varietà di linee chiuse, la quale circonda il punto $O$ e tende al medesimo.

Nelle vicinanze dell' elemento $O$, quando in quest' ultimo cada un massimo o minimo relativo, la funzione $f(x, y)$ d̀ al certo di seconda e quarta specie oppure di prima e terza.

Infatti, se consideriamo una linea $f(x, y)=C$, racchiudente il punto $O$, avvertiremo tosto che essa contiene al certo una parte $C^{(1)}$ analoga all' altra $C_{A}{ }^{(1)}$ ed una seconda $C^{(3)}$ conforme all' elemento $C_{A}{ }^{(3)}$ oppure due enti della forma $C^{(2)}$ e $C^{(4)}\left({ }^{*}\right)$. Stando la prima ipotesi io posso torre dalla linea $C^{(1)}$ un tratto connesso $p_{*}$ che cade per intero entro una delle aree $B_{\sigma}$ ed altrettanto si dica dell' elemento $q_{\tau}$ rispetto alla linea $C^{(3)}$. I tratti $p_{s}$ e $q_{\tau}$ appartengono per ordine ad una funzione di prima e terza specie o viceversa, secondo che il punto $O$ è un minimo od un massimo. In maniera analoga si direbbe delle linee $C^{(2)}$ e $C^{(4)}$.

$\dot{\mathrm{E}}$ chiaro che dal punto $O$ potrebbero uscire anche dei rami in ciascuno dei quali la $f(x, y)$ non muta di valore, ecco due esempi

$$
\underset{o}{\mid \mathrm{IV}, \mathrm{II}, \mathrm{I}} ;\left.\quad \underline{\mathrm{I}, \mathrm{III}}{ }_{0} \quad \overline{\mathrm{III}, \mathrm{IV}, \mathrm{III}}\right|_{m} ^{o} \quad \underset{m}{\mid \mathrm{III}, \mathrm{IV}} .
$$

Nel primo quadrante fra l'elemento IV e l'ente II vi è un ramo primitivo lungo il quale $f(x, y)=$ cost., nè un fatto simile ha luogo altrove. Ogni punto interno al ramo indicato è un minimo relativo della nostra funzione. Dall' ente $O$ poi escono altre due linee $f(x, y)=C$ (I $|, \underline{\mathrm{III}}|)$.

Il secondo esempio è:

$$
\underset{o}{\mid \mathrm{II}, \mathrm{IV}} ; \quad \underline{\mathrm{IV}, \mathrm{I}_{0}^{M}} ;\left.\quad \overline{\mathrm{I}, \mathrm{II} \mid}\right|_{m} ^{o} \quad{ }_{\mid \overline{\mathrm{II}} .}^{o}
$$

Nel primo quadrante tra gli enti II e IV havvi un ramo primitivo in cui $f(x, y)=$ cost., ogni punto interno del quale è un massimo della nostra funzione, mentre è altresi tale l'elemento $O$. Il modo di comportarsi delle linee $f(x, y)=$ cost. nei pressi di quest'ultimo è manifesto.

(*) Vedi la Memoria che precede, N. VI, pag. 1. 